\section{THE GENERATION OF SUM AND DIFFERENCE PATTERNS USING FRACTAL SUBARRAYS}

\section{H. Werner, ${ }^{1}$ K. C. Anushko, ${ }^{1}$ and P. L. Werner ${ }^{2}$}

${ }^{1}$ Applied Research Laboratory

The Pennsylvania State University

State College, Pennsylvania 16804-0030

${ }^{2}$ College of Engineering

The Pennsylvania State University

DuBois, Pennsylvania 15801

\section{Received 12 January 1999}

ABSTRACT: In this letter, we explore the potential for the application of fractal subarrays to the generation of sum and difference patterns. For the purposes of this investigation, a standard planar array is decomposed into two subarrays: one in the form of a Sierpinski carpet, and the other consisting of its complement. A methodology is then introduced for feeding the two subarrays in order to produce either a sum or a difference pattern. A particular example is considered in which directive gain plots are obtained for both the sum and difference modes of a $27 \times 27$ planar array. (c) 1999 John Wiley \& Sons, Inc. Microwave Opt Technol Lett 22: 54-57, 1999.

Key words: fractal antenna engineering; fractal antenna arrays; Sierpinski carpets; sum and difference modes

\section{INTRODUCTION}

Traditional approaches to the analysis and design of antenna systems have their foundation in Euclidean geometry. There has been a considerable amount of recent interest, however, in the possibility for developing new types of antennas which employ fractal rather than Euclidean geometric concepts in their design. This new area of research is known as fractal antenna engineering. Recent developments in the field of fractal antenna engineering are documented in [1-4]. For instance, an approach for designing low-sidelobe arrays has been proposed in [1], which is based on the theory of random fractals. The fact that self-scalable arrays generate fractal radiation patterns was first established in [2]. This work led to the development of a technique for synthesizing fractal radiation patterns that have a certain desired fractal dimension. The application of fractal concepts to the design of multiband Koch arrays as well as low-sidelobe Cantor arrays are discussed in [3]. A more general fractal geometric interpretation of classical frequency-independent antenna theory has been offered in [4]. Also introduced in [4] is a design methodology for multiband Weierstrass fractal arrays. The purpose of this letter is to investigate the radiation characteristics of a special type of fractal planar array whose elements form a Sierpinski carpet [5]. In particular, a new method will be outlined for generating sum and difference patterns which makes use of Sierpinski carpet fractal subarrays.

\section{THEORY}

Suppose we consider a generating array which has an array factor denoted by $G A(\psi)$. Then it is possible to construct a fractal array from this generator such that the corresponding array factor may be expressed in the compact product form $[6,7]$

$$
A F_{P}(\psi)=\prod_{p=1}^{P} G A\left(s^{p-1} \psi\right)
$$

where $P$ represents the stage of growth of the fractal array and $s$ is a scale factor that controls the rate of growth with each successive stage. One of the characteristic features of these fractal arrays is that they contain copies of the generating array at many different scales.

In this letter, we will consider a simple generator array of the form

$\begin{array}{lll}1 & 1 & 1 \\ 1 & 0 & 1 \\ 1 & 1 & 1\end{array}$

which represents a $3 \times 3$ planar array with the center element turned off or removed. It can be shown that, in this case, the generating array factor may be expressed as

$$
G A\left(\psi_{x}, \psi_{y}\right)=2\left[\cos \left(\psi_{x}\right)+\cos \left(\psi_{y}\right)+2 \cos \left(\psi_{x}\right) \cos \left(\psi_{y}\right)\right]
$$

where

$$
\begin{aligned}
\psi_{x} & =k d_{x} u_{s x} \\
\psi_{y} & =k d_{y} u_{s y} \\
u_{s x} & =\sin \theta \cos \phi-\sin \theta_{s} \cos \phi_{s} \\
u_{s y} & =\sin \theta \sin \phi-\sin \theta_{s} \sin \phi_{s} \\
d_{x} & =\text { element spacing in the } x \text {-direction } \\
d_{y} & =\text { element spacing in the } y \text {-direction } \\
\left(\theta_{s}, \phi_{s}\right) & =\text { steering angles. }
\end{aligned}
$$

This represents the generating array factor for a certain Sierpinski carpet fractal array which has a corresponding array factor at scale $P$ given by

$$
\begin{array}{r}
A F_{P}\left(\psi_{x}, \psi_{y}\right)=2^{P} \prod_{p=1}^{P}\left[\cos \left(3^{p-1} \psi_{x}\right)+\cos \left(3^{p-1} \psi_{y}\right)\right. \\
\left.+2 \cos \left(3^{p-1} \psi_{x}\right) \cos \left(3^{p-1} \psi_{y}\right)\right] .
\end{array}
$$

Furthermore, if the spacing between array elements is chosen such that $d_{x}=d_{y}=\lambda / 2$, then $\psi_{x}=\pi u_{s x}$ and $\psi_{y}=\pi u_{s y}$.

At this point in the development, we make the observation that the sum of the Sierpinski carpet array together with its complement at any stage $P$ results in a full $N \times N$ planar array where $N=3^{P}$. This relationship may be represented in mathematical terms by the equation

$$
A F_{P}\left(\psi_{x}, \psi_{y}\right)+\overline{A F_{P}}\left(\psi_{x}, \psi_{y}\right)=A F\left(\psi_{x}, \psi_{y}\right)
$$

where $A F_{P}$ is the Sierpinski carpet array factor, $\overline{A F_{P}}$ is the array factor associated with its complement, and $A F$ denotes the array factor of the full planar array. A convenient representation for the array factor of the full array may be found, which is given by

$$
A F\left(\psi_{x}, \psi_{y}\right)=\left[1-2 f_{P}\left(\psi_{x}\right)\right]\left[1-2 f_{P}\left(\psi_{y}\right)\right]
$$


where

$$
f_{P}(x)=\frac{\cos \left[\frac{1}{4}\left(3^{P}-1\right) x\right] \sin \left[\frac{1}{4}\left(3^{P}+1\right) x\right]}{\sin \left[\frac{1}{2} x\right]} .
$$

Finally, by using (5) together with (3), an expression for the complementary array factor $\overline{A F_{P}}$ may be obtained directly from (4).

The procedure for decomposing a planar array into the sum of a Sierpinski carpet fractal subarray and its associated complement may be illustrated by considering the following example. Suppose we have a $27 \times 27$ planar array with element locations as shown in Figure 1. In this particular case, we find that the array contains a stage three $(P=3)$ Sierpinski carpet subarray. The elements belonging to this fractal subarray are denoted in Figure 1 by dots. The remaining subarray, whose elements are indicated by asterisks, is formed by taking the complement of the Sierpinski carpet subarray. In other words, it consists of every element of the full array which is not an element of the carpet.

We next construct sum and difference patterns from a linear combination of the Sierpinski carpet subarray and the subarray formed by its complement. These patterns are defined in the following way:

$$
\begin{aligned}
& \Sigma f=A F_{P}+\alpha_{P} \overline{A F_{P}} \\
& \Delta f=A F_{P}-\alpha_{P} \overline{A F_{P}}
\end{aligned}
$$

where $\Sigma f$ represents the sum pattern, $\Delta f$ represents the difference pattern, and $\alpha_{P}$ is a positive real-valued constant. The directive gain associated with the sum and difference modes of such an array may be found using (7) and (8), respectively. The resulting expressions are

$$
\begin{aligned}
& D_{\Sigma}=\frac{4 \pi(\Sigma f)^{2}}{\int_{0}^{2 \pi} \int_{0}^{\pi}(\Sigma f)^{2} \sin \theta d \theta d \phi} \\
& D_{\Delta}=\frac{4 \pi(\Delta f)^{2}}{\int_{0}^{2 \pi} \int_{0}^{\pi}(\Delta f)^{2} \sin \theta d \theta d \phi} .
\end{aligned}
$$

A methodology is now introduced for making the proper choice of $\alpha_{P}$. The first step in this procedure is to recognize that the difference mode requires $D_{\Delta}=0$ when $\theta=\theta_{s}=0^{\circ}$. An inspection of (10) suggests that this condition will be satisfied if

$$
\Delta f=0, \quad \text { when } \theta=\theta_{s}=0^{\circ} .
$$

Substituting (8) into (11) and solving for $\alpha_{P}$ leads to

$$
\alpha_{P}=\left.\frac{A F_{P}}{\overline{A F_{P}}}\right|_{\theta=\theta_{s}=0^{\circ}} .
$$

It follows directly from (3) that

$$
\left.A F_{P}\right|_{\theta=\theta_{s}=0^{\circ}}=8^{P} .
$$

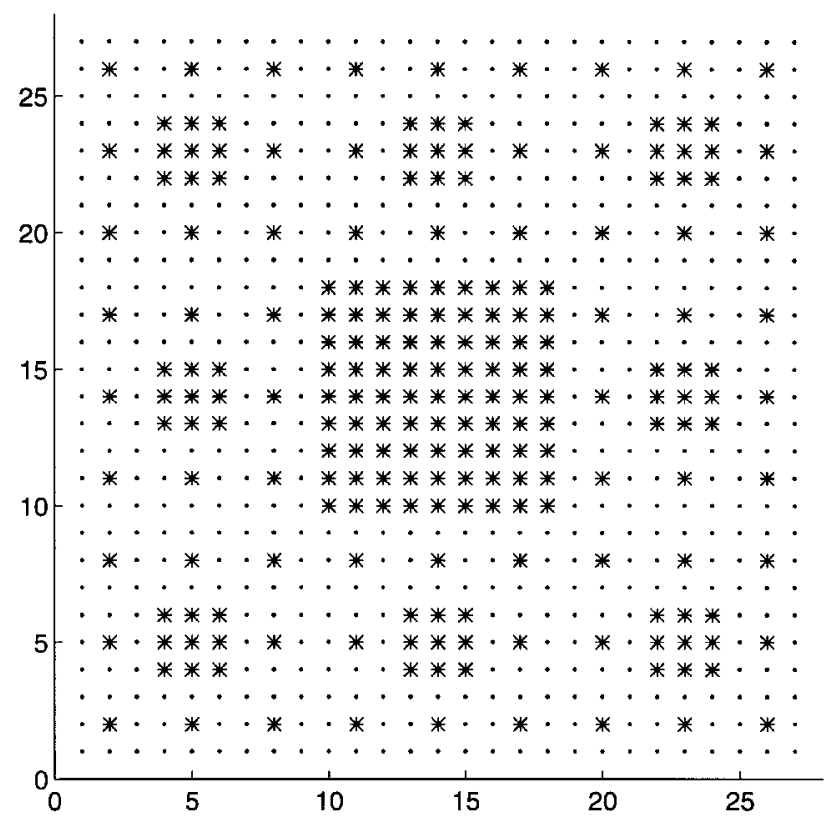

Figure 1 Elements in a full $27 \times 27$ planar array. The elements denoted by " $\bullet$ " are elements in the Sierpinski carpet subarray, and the elements denoted by "*" are elements in the complement subarray

Likewise, (5) and (6) may be used to show that

$$
\left.A F\right|_{\theta=\theta_{s}=0^{\circ}}=9^{P} .
$$

A useful formula for $\alpha_{P}$ may be found by combining (4), (13), and (14) together with (12). The simple design equation which results is

$$
\alpha_{P}=\frac{1}{(9 / 8)^{P}-1} .
$$

The first four values of $\alpha_{P}$ and the corresponding array size for each stage $P$ are listed in Table 1 .

The above development suggests that if the element excitation currents in the Sierpinski carpet subarray are

$$
I_{m n}=I_{0}, \quad \text { for } m n \in \text { carpet, }
$$

then the currents in the subarray formed by the complement of the carpet must be

$$
I_{m n}= \pm \alpha_{P} I_{0}, \quad \text { for } m n \in \text { complement }
$$

where the positive sign is for the sum mode $(\Sigma)$, the negative sign is for the difference mode $(\Delta)$, and $m, n=1,2, \ldots, 3^{P}$. Finally, it follows directly from (7), (12), and (13) that

$$
\Sigma f=2\left(8^{P}\right), \quad \text { when } \theta=\theta_{s}=0^{\circ} .
$$

\section{RESULTS}

In this section, we consider the sum and difference modes corresponding to the $27 \times 27$ planar array shown in Figure 1 . The directive gain pattern produced by operating the array in the sum mode is shown in Figure 2, while Figure 3 illustrates the pattern associated with the difference mode. In Figure 3, we see the deep null at $\theta=0^{\circ}$ that is created in the difference pattern by the correct choice of $\alpha_{3}$ (see Table 1). A plot of the directive gain for the full $27 \times 27$ array with a uniform 
TABLE 1 Tabulated Values of $\alpha$ as a Function of $P$

\begin{tabular}{llc}
\hline$P$ & \multicolumn{1}{c}{$\alpha$} & Array Size \\
\hline 1 & 8 & $3 \times 3$ \\
2 & 3.7647 & $9 \times 9$ \\
3 & 2.3594 & $27 \times 27$ \\
4 & 1.6617 & $81 \times 81$ \\
\hline
\end{tabular}

current excitation (i.e., $I_{m n}=1$ for all values of $m$ and $n$ ) is shown in Figure 4 for comparison purposes.

\section{CONCLUSIONS}

A technique has been introduced in this letter for generating sum and difference patterns using fractal subarrays. The particular type of fractal subarrays which were investigated

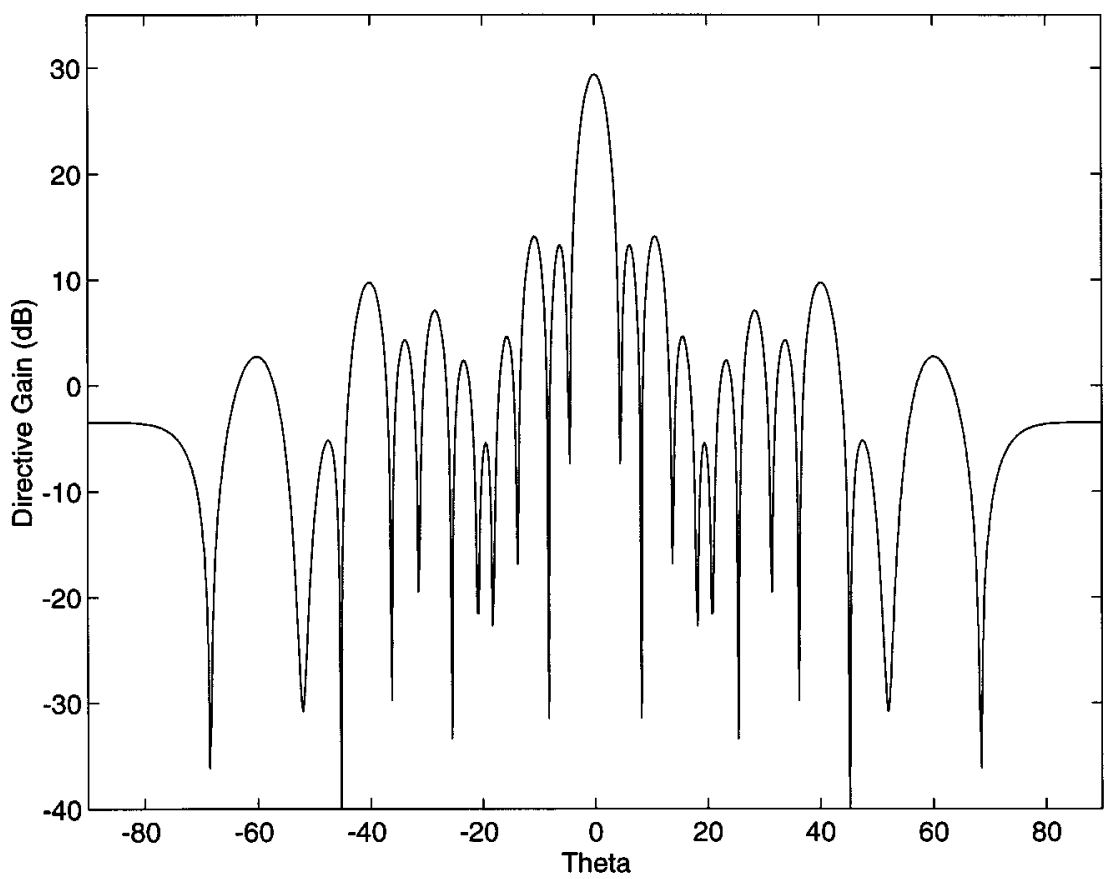

Figure 2 Directive gain plot for the sum mode of the $27 \times 27$ array shown in Figure 1. Spacing between the elements in the array is held constant at $d=\lambda / 2$ with $\phi=0^{\circ}$. The maximum directive gain for the sum mode of stage 3 is $D_{3}=29.36 \mathrm{~dB}$

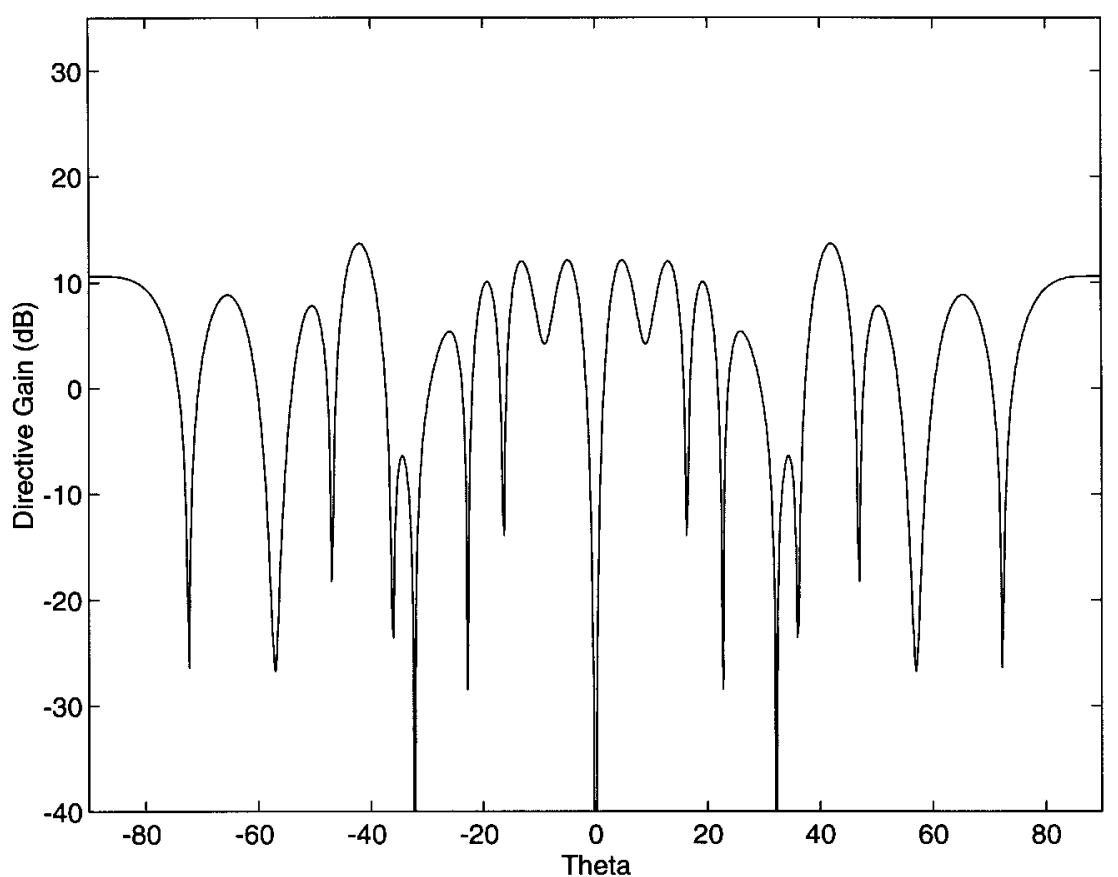

Figure 3 Directive gain plot for the difference mode of the $27 \times 27$ array shown in Figure 1 . Spacing between the elements in the array is held constant at $d=\lambda / 2$ with $\phi=0^{\circ}$. The maximum directive gain for the difference mode of stage 3 is $D_{3}=13.70 \mathrm{~dB}$, with a deep null appearing at $\theta=0^{\circ}$ 


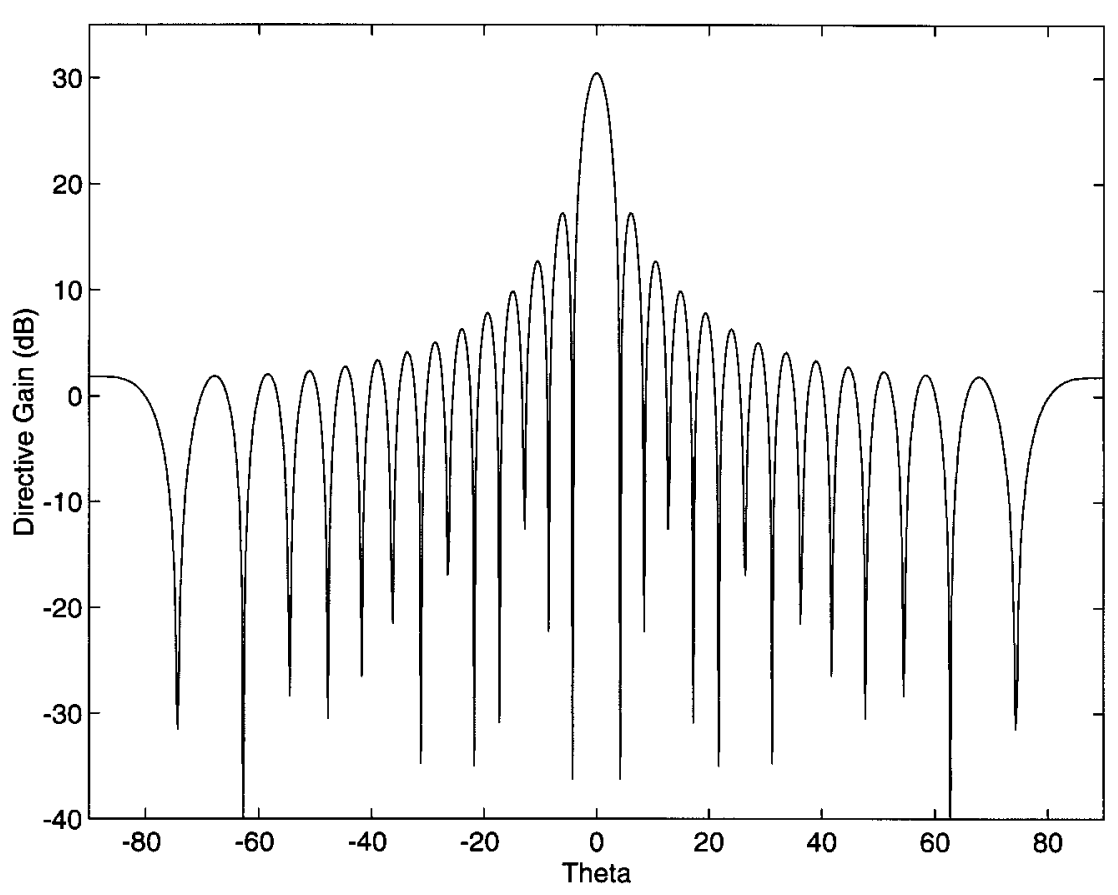

Figure 4 Directive gain plot for the full $27 \times 27$ planar array. Spacing between the elements in the array is held constant at $d=\lambda / 2$ with $\phi=0^{\circ}$. The maximum directive gain for this array is $D=30.46 \mathrm{~dB}$

belong to the family of Sierpinski carpets. An example was considered in which sum and difference patterns were generated using a $27 \times 27$ planar array that contained a stage-three Sierpinski carpet fractal subarray.

\section{REFERENCES}

1. Y. Kim and D.L. Jaggard, The fractal random array, Proc IEEE 44 (1986), 1278-1280.

2. D.H. Werner and P.L. Werner, On the synthesis of fractal radiation patterns, Radio Sci 30 (1995), 29-45.

3. C. Puente-Baliarda and R. Pous, Fractal design of multiband and low side-lobe arrays, IEEE Trans Antennas Propagat 44 (1996), 730-739.

4. D.H. Werner and P.L. Werner, Frequency-independent features of self-similar fractal antennas, Radio Sci 31 (1996), 1331-1343.

5. H.-O. Peitgen, H. Jürgens, and D. Saupe, Chaos and fractals: New frontiers of science, Springer-Verlag, New York, 1992, Chap. 2, p. 81.

6. R.L. Haupt and D.H. Werner, Fast array factor calculations for fractal arrays, Proc 13th Annu Rev of Progress in Applied Computational Electromagn (ACES), Monterey, CA, 1997, vol. 1, pp. 291-296.

7. D.H. Werner and R.L. Haupt, Fractal constructions of linear and planar arrays, Proc IEEE Antennas Propagat Soc Int Symp, Montreal, Canada, 1997, vol. 3, pp. 1968-1971.

(c) 1999 John Wiley \& Sons, Inc.

CCC 0895-2477/99

\section{DESIGN AND MODELING USING THE FDTD METHOD OF PLANAR MULTIAPPLICATORS FOR MICROWAVE HYPERTHERMIA}

\author{
P.-Y. Cresson, ${ }^{1}$ L. Dubois, ${ }^{1}$ M. Chive, ${ }^{1}$ and J. Pribetich ${ }^{1}$ \\ ${ }^{1}$ Département Hyperfréquences et Semiconducteurs \\ IEMN — UMR CNRS 9929 \\ Université des Sciences et Technologies de Lille \\ 59652 Villeneuve d'Ascq Cedex, France
}

\section{Received 16 December 1998}

ABSTRACT: This paper describes a new kind of planar applicator called the multiapplicator which has been developed for external microwave hyperthermia controlled by microwave radiometry. The possibility to obtain larger heating patterns than with other applicators with several patches is clearly focused by the theoretical results which are presented and verified by experimental measurements. (c) 1999 John Wiley \& Sons, Inc. Microwave Opt Technol Lett 22: 57-63, 1999.

Key words: microwave hyperthermia; planar multiapplicator; microwave heating; microwave antennas

\section{INTRODUCTION}

One of the main objectives of using microwaves in hyperthermia for cancer treatment is to develop the capability of delivering therapeutic heat to tumors without overheating the surrounding healthy tissues. To achieve microwave hyperthermia, specific applicators [1-4] have been designed according to the anatomic locations of tumors or tissues to be heated. Among these devices, we have developed, for more than a decade, planar external applicators which present several advantages: small size, light weight, and capable of conforming to the shape of the body. Further, they are effective in coupling energy directly into tissues with minimal stray fields. They are generally used for the treatment of deep- or semideep-seated tumors. 